\title{
ORTHOGONAL POLYNOMIALS ASSOCIATED WITH INVARIANT MEASURES ON JULIA SETS
}

\author{
BY M. F. BARNSLEY ${ }^{1}$, J. S. GERONIMO ${ }^{2}$ AND A. N. HARRINGTON
}

I. Introduction. Let $\mathbf{C}$ be the complex plane and $T: \mathbf{C} \rightarrow \mathbf{C}$ be a nonlinear polynomial of the form $T(z)=z^{n}+k_{1} z^{n-1}+\cdots+k_{n}$. Consider the sequence $\left\{T^{n}(z)\right\}, n=1,2, \ldots$, where $T^{0}(z)=z$ and $T^{n}(z)=T \circ T^{n-1}(z)$. The general theory of sequences of this form has been developed by Fatou $[5,6]$, Julia [8] and Brolin [4]. In their research a fundamental role is played by the Julia set $B$, which is the set of points in $\mathbf{C}$ where $\left\{T^{n}(z)\right\}$ is not normal.

Fatou and Julia established the possible structures $B$ can have and showed that these depend in a complicated way on the coefficients of $T(z)$. Among other things they demonstrated that $B$ may be the unit circle, a straight line, a generalized Cantor set, or a set containing an infinite number of Jordan curves. However, in all cases, $B$ is compact and $T^{-1}(B)=B$.

In 1965 Brolin established some electrical properties of the set $B$. He showed that the logarithmic capacity of $B$ is positive and that there exists an equilibrium charge distribution $u$. He also proved that, for $T: B \rightarrow B, u$ is invariant and the system $(B, u, T)$ is strongly mixing. It is the purpose of this note to develop more fully the properties of the equilibrium measure on $B$ and to investigate the monic polynomials orthogonal with respect to this measure.

II. Results. We begin with the following

Definition 1. $u$ is a balanced $T$-invariant measure on $B$ if $u$ is a probability measure supported on $B$ such that for any complete assignment of branches of $T^{-1}, T_{j}^{-1}, j=1,2, \ldots, n, u\left(T_{j}^{-1}(S)\right)=u(S) / n$ for each Borel set $S$.

REMARK 1. One can show [2] that there is only one balanced $T$-invariant measure on $B$ and that the measure constructed by Brolin is balanced.

Received by the editors January 28, 1982.

1980 Mathematics Subject Classification. Primary 42 A52.

${ }^{1}$ Partially supported by N.S.F. Grant MCS-8104862.

${ }^{2}$ Partially supported by N.S.F. Grant MCS-8002731. 
For any $T$-invariant measure one has $\int_{B} f d u=\int_{B} f \circ T d u$, however if $u$ is balanced one has

THEOREM 1. Let $u$ be the balanced T-invariant measure on $B$, and $f \in$ $L^{1}(B, u)$, then

$$
\frac{1}{n} \int_{B} \sum_{k=1}^{n} f\left(T_{k}^{-1}(z)\right) d u(z)=\int_{B} f(z) d u(z) .
$$

Proof. It is sufficient to take $f$ to be a characteristic function $\chi_{S}$ for an arbitrary $u$-measurable set $S \subset T_{j}^{-1}(B)$. With these assumptions one has $T_{k}^{-1}(B)$ $\cap S, k \neq j$, contains only a countable number of points and $T_{j}^{-1}(x) \in S \Leftrightarrow$ $x \in T(S)$. Therefore

$$
\frac{1}{n} \int_{B} \sum_{k=1}^{n} \chi_{S}\left(T_{k}^{-1}(z)\right) d u(z)=\frac{1}{n} \int_{B} \chi_{S}\left(T_{j}^{-1}(z)\right) d u(z)=\frac{1}{n} u(T(S)) .
$$

Now using the fact that $u$ is balanced and then the assumption on $S$ one has

$$
\frac{1}{n} u(T(S))=u\left(T_{j}^{-1}(T(S))\right)=u(S)=\int_{B} \chi_{S} d u
$$

Before continuing on to the next theorem we review some algebraic facts about the polynomial $P(z)=T(z)-c$, where $c$ is a complex variable. Set $s_{m}=$ $\sum_{l=1}^{n}\left(T_{l}^{-1}(c)\right)^{m}, m=1,2, \ldots, n-1$. It is well known (Gaal [7, pp. 24-25]), that $s_{m}=-m k_{m}-\sum_{l=1}^{m-1} k_{l} s_{m-l}$ where $k_{j}$ is the coefficient of $z^{n-j}$ in $P$ and is independent of $c$ for $j=1,2, \ldots, n-1$. Thus the value of $s_{m}$ depends only on the polynomial $T$ and not on $c$. This leads to

THEOREM 2. Let $u$ be the balanced T-invariant measure on $B$, and let $f$ $f \in L^{1}(B, u)$. Then

$$
\int_{B} z^{j} f(T(z)) d u(z)=\frac{s_{j}}{n} \int_{B} f(z) d u(z) \quad \text { for } j=1,2, \ldots, n-1
$$

Proof. Applying Theorem 1 yields

$$
\begin{aligned}
\int_{B} z^{j} f(T(z)) d u(z) & =\int_{B} \frac{1}{n} \sum_{k=1}^{n}\left(T_{k}^{-1}(z)\right)^{j} f(z) d u(z) \\
& =\frac{s_{j}}{n} \int_{B} f(z) d u(z) \text { for } j=1,2, \ldots, n-1
\end{aligned}
$$

One now has the following relations among the monic orthogonal polynomials associated with $u$. 
THEOREM 3. Let $u$ be the balanced T-invariant measure on $B$. Let $\left\{P_{l}\right\}$ be the sequence of monic polynomials orthogonal with respect to $u$, with $P_{l}$ of degree l, satisfying $\int_{B} P_{n}(z) \overline{P_{m}(z)} d u(z)=0, n \neq m$, then

(1) $P_{1}(z)=z+k_{1} / n$.

(2) $P_{l n}(z)=P_{l}(T(z))$ for $l=0,1,2, \ldots$.

(3) $P_{n l}(z)=T^{l}(z)+k_{1} / n$ for $l=0,1,2, \ldots$

Proof. (1) is proved by integrating $P_{1}(z)$ and using Theorem 2. To prove (2) it is sufficient to show that $P_{l}(T(z))$ is orthogonal to polynomials of the form $z^{j}(T(z))^{m}, j=0,1,2, \ldots, n-1 ; m=0,1, \ldots, l-1$. Denoting conjugation by - and applying Theorem 2 to the following integral yields

$$
\int_{B} z^{j}(T(z))^{m} \overline{P_{l}(T(z))} d u(z)=\frac{s_{j}}{n} \int_{B} z^{m} \overline{P_{l}(z)} d u(z)=0
$$

since $P_{l}(z)$ is an orthogonal polynomial of degree $l$. Finally (3) follows from (1) and (2) by induction.

REMARK 2. From the electrical interpretation of $B$ one can see that if $B$ is an interval $[a, b]$ on the real line then $d u(x)=\pi^{-1}((b-x)(x-a))^{-1 / 2} d x$ and $\left\{P_{l}(x)\right\}$ are the shifted Chebyshev polynomials associated with $d u$.

REMARK 3. Under the Mobius transformation $(z, T z) \rightarrow(R z, R T z)$ with $R(z)=z+k_{1} / n$ one can transform $T$ to a topologically equivalent $\widetilde{T}=z^{n}+$ $\widetilde{k}_{2} z^{n-2}+\cdots+\widetilde{k}_{n}$. In this case the iterates of $\widetilde{T}$ are monic orthogonal polynomials associated with $d \tilde{u}$.

Let $U$ and $D$ be the components of the complement of $B$, and the unit circle, respectively containing $\infty$. Fatou $[5,6]$ has shown that there is a function analytic at $\infty$, normalized so that $F(z)=z+O(1)$, which obeys the Böttcher functional equation

$$
F(T(z))=(F(z))^{n}
$$

$F$ may be extended to be a well-defined analytic function in any simply-connected subregion of $U$.

THEOREM 4.

$$
\left(P_{n k}(z)\right)^{1 / n^{k}}=F(z)\left(1+O\left(z^{-2 n^{k}}\right)\right)
$$

and the $\left[n^{k}-1 / n^{k}\right]$ Pade approximant to the moment generating function

$$
G(z)=\int_{B} \frac{d u(x)}{1-z x} \text { is } \frac{P_{n k}^{\prime}(1 / z) / z}{P_{n k}(1 / z) n^{k}}
$$

SKETCH OF THE PROOF. Let $F(z)=z+b_{0}+b_{1} / z+\cdots$. Then from electrical considerations one has

$$
\log F(z)=\int_{B} \log (z-x) d u(x) .
$$


Expanding the above equation yields $b_{0}=-\int_{B} x d u(x)=k_{1} / n$. From the iteration of (4) one obtains $F(z)=\left(F\left(T^{k}(z)\right)\right)^{1 / n^{k}}$. (5) can now be retrieved by substituting in the power series for $F$ and using equation (1). The Pade formula follows from differentiation using (5) and (6) and replacing $z$ by $1 / z$.

REMARK 6. If $B$ is connected, $F$ maps $U$ conformally onto $D$. An equation related to (5) for polynomials orthogonal with respect to a measure on a rectifiable Jordan curve is proved in Szegö [10, p. 372], (see also Smirnoff [9]). However, as noted earlier, $B$ may contain an infinite number of Jordan curves or be disconnected.

REMARK 7. It follows from Brolin's construction of $u$ that the poles of our Padé approximants become dense on $B$ in the limit as $n^{k} \rightarrow \infty$. This is of considerable interest to Padé approximant theory because cases where one has total pole control for an infinite subsequence are desirable but not common: examples are usually restricted to measures which are supported on the real line.

The special case when $T$ is quadratic has been investigated by Barnsley et al [1] and Bessis et al [3].

Further considerations of the electrical properties, as well as more detailed results concerning the orthogonal polynomials and the three term recurrence relation satisfied by them when the measure is supported on the real line, are given in Barnsley et al [2].

\section{REFERENCES}

1. M. F. Barnsley, J. S. Geronimo and A. N. Harrington, On the invariant sets of a family of quadratic maps, Comm. Math. Phys. (submitted).

2. M. F. Barnsley, J. S. Geronimo and A. N. Harrington (in preparation)

3. D. Bessis, M. L. Mehta, and P. Moussa, Orthogonal polynomials on a family of Cantor sets and the problem of iterations of quadratic mappings, Letters in Math. Phys. (submitted).

4. H. Brolin, Invariant sets under iteration of rational functions, Ark. Mat. 6 (1965), $103-144$. $161-271$

5. P. Fatou, Sur les équations fonctionelles, Bull. Soc. Math. France 47 (1919), $208-314$.

6. - Sur les équations fonctionelles, Bull. Soc. Math. France 48 (1920), 33-94;

7. L. Gaal, Classical Galois theory with examples, Markham, Chicago, 1971.

8. G. Julia, Memoire sur L'iteration des fonctions rationelles, J. Math. Pures Appl. Ser. 81 (1918), 47-245.

9. V. J. Smirnoff, Sur les formules de Cauchy et de Green quelques problemes qui s'y rattachent, Bull. Acad. Sci. URSS (1932), 337-372.

10. G. Szegö, Orthogonal polynomials, Amer. Math. Soc. Colloq. Publ., vol. 23, Amer. Math. Soc., Providence, RI, 1939.

SCHOOL OF MATHEMATICS, GEORGIA INSTITUTE OF TECHNOLOGY, ATLANTA, GEORGIA 30332 